M. Aprodu and V. Brînzănescu

Nagoya Math. J.

Vol. 154 (1999), 111-122

\title{
MODULI SPACES OF VECTOR BUNDLES OVER RULED SURFACES
}

\author{
MARIAN APRODU AND VASILE BRÎNZǍNESCU
}

\begin{abstract}
We study moduli spaces $M\left(c_{1}, c_{2}, d, r\right)$ of isomorphism classes of algebraic 2-vector bundles with fixed numerical invariants $c_{1}, c_{2}, d, r$ over a ruled surface. These moduli spaces are independent of any ample line bundle on the surface. The main result gives necessary and sufficient conditions for the nonemptiness of the space $M\left(c_{1}, c_{2}, d, r\right)$ and we apply this result to the moduli spaces $\mathcal{M}_{L}\left(c_{1}, c_{2}\right)$ of stable bundles, where $L$ is an ample line bundle on the ruled surface.
\end{abstract}

\section{Introduction}

Let $\pi: X \rightarrow C$ be a ruled surface over a smooth algebraic curve $C$, defined over the complex number field $\mathbb{C}$. Let $f$ be a fibre of $\pi$. Let $c_{1} \in \operatorname{Num}(X)$ and $c_{2} \in H^{4}(X, \mathbb{Z}) \cong \mathbb{Z}$ be fixed. For any polarization $L$, denote the moduli space of rank-2 vector bundles stable with respect to $L$ in the sense of Mumford-Takemoto by $\mathcal{M}_{L}\left(c_{1}, c_{2}\right)$. Stable 2-vector bundles over a ruled surface have been investigated by many authors; see, for example [T1], [T2], [H-S], [Q1]. Let us mention that Takemoto [T1] showed that there is no rank-2 vector bundle (having $c_{1} . f$ even) stable with respect to every polarization $L$. In this paper we shall study algebraic 2-vector bundles over ruled surfaces, but we adopt another point of view: we shall study moduli spaces of (algebraic) 2-vector bundles over a ruled surface $X$, which are defined independent of any ample divisor (line bundle) on $X$, by taking into account the special geometry of a ruled surface (see [B], [B-St1], [B-St2] and also [Br1], [Br2], [W]).

In Section 1 (put for the convenience of the reader) we present (see [B]) two numerical invariants $d$ and $r$ for a 2-vector bundle with fixed Chern classes $c_{1}$ and $c_{2}$ and we define the set $M\left(c_{1}, c_{2}, d, r\right)$ of isomorphism classes of bundles with fixed invariants $c_{1}, c_{2}, d, r$. The integer $d$ is given by the splitting of the bundle on the general fibre and the integer $r$ is given by some normalization of the bundle. Recall that the set $M\left(c_{1}, c_{2}, d, r\right)$ carries

Received February 8, 1996. 
a natural structure of an algebraic variety (see [B], [B-St1], [B-St2]). In Section 2 we study uniform vector bundles and we prove the existence of algebraic vector bundles given by extensions of line bundles and which are not uniform. In Section 3 the main result gives necessary and sufficient conditions for the non-emptiness of the space $M\left(c_{1}, c_{2}, d, r\right)$ and we apply this result to the moduli space of stable bundles $\mathcal{M}_{L}\left(c_{1}, c_{2}\right)$.

\section{$\S 1$. Moduli spaces of rank-2 vector bundles}

In this section we shall recall from ([B], [B-St1], [B-St2]) some basic notions and facts.

The notations and the terminology are those of Hartshorne's book [Ha]. Let $C$ be a nonsingular curve of genus $g$ over the complex number field and let $\pi: X \rightarrow C$ be a ruled surface over $C$. We shall write $X \cong \mathbb{P}(\mathcal{E})$ where $\mathcal{E}$ is normalized. Let us denote by e the divisor on $C$ corresponding to $\bigwedge^{2} \mathcal{E}$ and by $e=-\operatorname{deg}(\mathbf{e})$. We fix a point $p_{0} \in C$ and a fibre $f_{0}=\pi^{-1}\left(p_{0}\right)$ of $X$. Let $C_{0}$ be a section of $\pi$ such that $\mathcal{O}_{X}\left(C_{0}\right) \cong \mathcal{O}_{\mathbb{P}(\mathcal{E})}(1)$.

Any element of $\operatorname{Num}(X) \cong H^{2}(X, \mathbb{Z})$ can be written $a C_{0}+b f_{0}$ with $a, b \in \mathbb{Z}$. We shall denote by $\mathcal{O}_{C}(1)$ the invertible sheaf associated to the divisor $p_{0}$ on $C$. If $L$ is an element of $\operatorname{Pic}(C)$ we shall write $L=\mathcal{O}_{C}(k) \otimes L_{0}$, where $L_{0} \in \operatorname{Pic}_{0}(C)$ and $k=\operatorname{deg}(L)$. We also denote by $F\left(a C_{0}+b f_{0}\right)=$ $F \otimes \mathcal{O}_{X}(a) \otimes \pi^{*} \mathcal{O}_{C}(b)$ for any sheaf $F$ on $X$ and any $a, b \in \mathbb{Z}$.

Let $E$ be an algebraic rank-2 vector bundle on $X$ with fixed numerical Chern classes $c_{1}=(\alpha, \beta) \in H^{2}(X, \mathbb{Z}) \cong \mathbb{Z} \times \mathbb{Z}, c_{2}=\gamma \in H^{4}(X, \mathbb{Z}) \cong \mathbb{Z}$, where $\alpha, \beta, \gamma \in \mathbb{Z}$.

Since the fibres of $\pi$ are isomorphic to $\mathbb{P}^{1}$ we can speak about the generic splitting type of $E$ and we have $\left.E\right|_{f} \cong \mathcal{O}_{f}(d) \oplus \mathcal{O}_{f}\left(d^{\prime}\right)$ for a general fibre $f$, where $d^{\prime} \leq d, d+d^{\prime}=\alpha$. The integer $d$ is the first numerical invariant of $E$.

The second numerical invariant is obtained by the following normalization:

$$
-r=\inf \left\{l \mid \exists L \in \operatorname{Pic}(C), \operatorname{deg}(L)=l \text {, s.t. } H^{0}\left(X, E\left(-d C_{0}\right) \otimes \pi^{*} L\right) \neq 0\right\} .
$$

We shall denote by $M(\alpha, \beta, \gamma, d, r)$ or $M\left(c_{1}, c_{2}, d, r\right)$ or $M$ the set of isomorphism classes of algebraic rank- 2 vector bundles on $X$ with fixed Chern classes $c_{1}, c_{2}$ and invariants $d$ and $r$.

With these notations we have the following result (see $[\mathrm{B}]$ ): 
TheOREm 1. For every vector bundle $E \in M\left(c_{1}, c_{2}, d, r\right)$ there exist $L_{1}, L_{2} \in \operatorname{Pic}_{0}(C)$ and $Y \subset X$ a locally complete intersection of codimension 2 in $X$, or the empty set, such that $E$ is given by an extension

$$
0 \rightarrow \mathcal{O}_{X}\left(d C_{0}+r f_{0}\right) \otimes \pi^{*} L_{2} \rightarrow E \rightarrow \mathcal{O}_{X}\left(d^{\prime} C_{0}+s f_{0}\right) \otimes \pi^{*} L_{1} \otimes I_{Y} \rightarrow 0,
$$

where $c_{1}=(\alpha, \beta) \in \mathbb{Z} \times \mathbb{Z}, c_{2}=\gamma \in \mathbb{Z}, d+d^{\prime}=\alpha, d \geq d^{\prime}, r+s=\beta$, $l\left(c_{1}, c_{2}, d, r\right):=\gamma+\alpha(d e-r)-\beta d+2 d r-d^{2} e=\operatorname{deg}(Y) \geq 0$.

Remark. By applying Theorem 1 we can obtain the canonical extensions used in [Br1], [Br2].

Indeed, let us suppose first that $d>d^{\prime}$. From the exact sequence (1) it follows that

$$
\mathcal{O}_{C}(r) \otimes L_{2} \cong \pi_{*} E\left(-d C_{0}\right)
$$

so

$$
\mathcal{O}_{X}\left(r f_{0}\right) \otimes \pi^{*} L_{2} \cong \pi^{*} \pi_{*} E\left(-d C_{0}\right)
$$

and

$$
\mathcal{O}_{X}\left(d C_{0}+r f_{0}\right) \otimes \pi^{*} L_{2} \cong\left(\pi^{*} \pi_{*} E\left(-d C_{0}\right)\right)\left(d C_{0}\right) .
$$

If $d=d^{\prime}$ then, by applying $\pi_{*}$ to the short exact sequence

$$
0 \rightarrow \mathcal{O}_{X}\left(r f_{0}\right) \otimes \pi^{*} L_{2} \rightarrow E\left(-d C_{0}\right) \rightarrow \mathcal{O}_{X}\left(s f_{0}\right) \otimes \pi^{*} L_{1} \otimes I_{Y} \rightarrow 0
$$

it follows the exact sequence

$$
0 \rightarrow \mathcal{O}_{C}(r) \otimes L_{2} \rightarrow \pi_{*} E\left(-d C_{0}\right) \rightarrow \mathcal{O}_{C}(s) \otimes L_{1} \otimes \mathcal{O}_{C}\left(-Z_{1}\right) \rightarrow 0,
$$

where $Z_{1}$ is an effective divisor on $C$ with the support $\pi(Y)$. With the notation $Z=\pi^{-1}\left(Z_{1}\right)$, by applying $\pi^{*}$ ( $\pi$ is a flat morphism) we obtain the following commutative diagram with exact rows

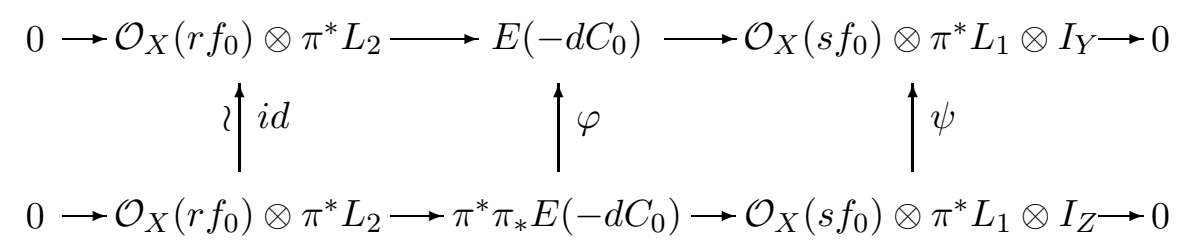


From the injectivity of $\psi$ we obtain the injectivity of $\varphi$. Because of

$$
\mathcal{O}_{X}\left(s f_{0}\right) \otimes \pi^{*} L_{1} \otimes I_{Y \subset Z} \cong \operatorname{Coker} \psi \cong \operatorname{Coker} \varphi
$$

we conclude.

Recall that a set $M$ of vector bundles on a $\mathbb{C}$-scheme $X$ is called bounded if there exists an algebraic $\mathbb{C}$-scheme $T$ and a vector bundle $V$ on $T \times X$ such that every $E \in M$ is isomorphic with $V_{t}=\left.V\right|_{t \times X}$ for some closed point $t \in T$ (see $[\mathrm{K}]$ ).

For the next result see $[\mathrm{B}]$ :

TheOREM 2. The set $M\left(c_{1}, c_{2}, d, r\right)$ is bounded.

\section{$\S 2$. Uniform bundles}

In what follows, we keep the notations from Section 1.

Definition 3. A 2-vector bundle $E$ is called an uniform bundle if the splitting type is preserved on all fibres of $X$.

Theorem 1 allows us to give a criterion for uniformness.

LEMMA 4. Let $f$ be a fibre of $X$ and let us suppose that $I_{Y \cap f \subset f} \cong$ $\mathcal{O}_{f}(-n)$. Then $\left.E\right|_{f} \cong \mathcal{O}_{f}(d+n) \oplus \mathcal{O}_{f}\left(d^{\prime}-n\right)$.

Proof. We suppose that $\left.E\right|_{f} \cong \mathcal{O}_{f}(a) \oplus \mathcal{O}_{f}\left(a^{\prime}\right)$, where $a \geq a^{\prime}$. Then we have a surjective morphism

$$
\left.E\right|_{f} \rightarrow \mathcal{O}_{f}\left(d^{\prime}\right) \otimes I_{Y} \otimes \mathcal{O}_{f}
$$

in virtue of Theorem 1 . On the other hand, the restriction of the sequence

$$
0 \rightarrow I_{Y} \rightarrow \mathcal{O}_{X} \rightarrow \mathcal{O}_{Y} \rightarrow 0
$$

to $f$ gives a surjective morphism

$$
I_{Y} \otimes \mathcal{O}_{f} \rightarrow I_{Y \cap f \subset f} \cong \mathcal{O}_{f}(-n) .
$$

So, we obtain another surjective morphism

$$
\mathcal{O}_{f}(a) \oplus \mathcal{O}_{f}\left(a^{\prime}\right) \rightarrow \mathcal{O}_{f}\left(d^{\prime}-n\right) .
$$

By using the inequalities $a \geq a^{\prime}, d \geq d^{\prime} \geq d^{\prime}-n$ and the equality $a+a^{\prime}=$ $d+d^{\prime}=\alpha$ it follows that $a^{\prime}=d^{\prime}-n$ and $a=d+n$. 
Corollary 5. E is an uniform bundle if and only if $l\left(c_{1}, c_{2}, d, r\right)=0$.

By means of Corollary 5 the uniform bundles are given by extensions of line bundles. It is naturally to ask if the converse is true. Unfortunately, this question has a negative answer, as proved by the following

Proposition 6. On the rational ruled surface $\mathbb{F}_{e}$ with $e \geq 1$ there exist non-uniform bundles given by extensions of line bundles.

For the proof we need some preparations.

Let $E$ be a 2-vector bundle given by an extension

$$
0 \rightarrow F \rightarrow E \rightarrow G \rightarrow 0
$$

where $F=\mathcal{O}_{X}\left(a C_{0}+r^{\prime} f_{0}\right) \otimes \pi^{*} L_{2}^{\prime}, G=\mathcal{O}_{X}\left(a^{\prime} C_{0}+s^{\prime} f_{0}\right) \otimes \pi^{*} L_{1}^{\prime}\left(L_{1}^{\prime}, L_{2}^{\prime} \in\right.$ $\left.\operatorname{Pic}_{0}(C)\right)$ are line bundles on $X$. By means of Theorem $1, E$ sits also in a canonical extension (1). If $a \geq a^{\prime}$ then $E$ is obviously uniform. Then, we shall suppose that $a<a^{\prime}$.

LEMMA 7. With the above notations we have $d \leq a^{\prime}$.

Proof. Indeed, by the restriction of the sequence (2) to a general fibre $f$ we obtain a surjective morphism

$$
\mathcal{O}_{f}(d) \oplus \mathcal{O}_{f}\left(d^{\prime}\right) \rightarrow \mathcal{O}_{f}\left(a^{\prime}\right)
$$

If $d>a^{\prime}$, then it follows that $d^{\prime}=a^{\prime}$ which contradicts the inequalities $a<a^{\prime}, d \geq d^{\prime}\left(a+a^{\prime}=d+d^{\prime}\right)$.

LEMMA 8. If $d=a^{\prime}$ then $E$ is uniform.

Proof. Let $f$ be a fibre of $X$ such that the splitting type of $\left.E\right|_{f}$ is different from the generic splitting type of $E$. According to Lemma 4

$$
\left.E\right|_{f} \cong \mathcal{O}_{f}(d+n) \oplus \mathcal{O}_{f}\left(d^{\prime}-n\right),
$$

where $n>0$.

By the restriction of (2) to $f$ we obtain a surjective morphism

$$
\mathcal{O}_{f}(d+n) \oplus \mathcal{O}_{f}\left(d^{\prime}-n\right) \rightarrow \mathcal{O}_{f}(d) .
$$

Because of $d+n>d$ it follows $d^{\prime}-n=d$, contradiction. 
LEMMA 9. In the above hypotheses, if $d=a^{\prime}$, then $E \cong F \oplus G$.

Proof. Let us observe that we can suppose, without loss of generality, that $a=0$ and $r^{\prime}=0$ (by twisting the sequences (1) and (2) with $\left.\mathcal{O}_{X}\left(-a C_{0}-r^{\prime} f_{0}\right)\right)$. Then, it follows that $d=a^{\prime}=\alpha>0, s^{\prime}=\beta$ and $d^{\prime}=0$.

Therefore, the sequences (1) and (2) become:



The computation of $c_{2}(E)$ in $\left(1^{\prime}\right)$ gives $\operatorname{deg}(Y)=-\alpha s$. Moreover, by means of Lemma $8, \operatorname{deg}(Y)=0$, so $s=0$ (we supposed $\alpha>0$ ).

The homomorphism $\chi=\varphi \psi$ is non-zero, otherwise $\mathcal{O}_{X}\left(\alpha C_{0}+\beta f_{0}\right) \subset$ $\pi^{*}\left(L_{2}^{\prime}\right)$ (which would contradict the condition $\alpha>0$ ), so $L_{2}=L_{1}^{\prime}$ and $\chi$ is the multiplication by a $\lambda \in \mathbb{C}^{*}$, and the assertion follows.

In this moment, we are able to give the counter-example announced in Proposition 6 .

Proof of Proposition 6 . Let $G$ be $\mathcal{O}_{X}\left(2 C_{0}\right)$ and let $F$ be $\mathcal{O}_{X}$. Then:

$$
\operatorname{dim} H^{1}\left(G^{-1}\right)=e+1 \neq 0 .
$$

For $E$ given by an extension $\xi \in \operatorname{Ext}^{1}\left(G, \mathcal{O}_{X}\right)$, keeping the notations from Section 1, we have $d \leq 2$ (Lemma 7), $d \geq d^{\prime}, d+d^{\prime}=2$ and $r+s=0$.

There are only two possibilities:

(a) $d=2, d^{\prime}=0$, which implies $E \cong \mathcal{O}_{X} \oplus \mathcal{O}_{X}\left(2 C_{0}\right)$ (Lemma 9).

(b) $d=d^{\prime}=1$ and, in this case, in the canonical extension (1) of $E$, we have 


$$
\operatorname{deg}(Y)=d d^{\prime} e-d s-d^{\prime} r=e \geq 1 .
$$

By applying Corollary 5, all vector bundles given by non-zero extensions from $\operatorname{Ext}^{1}\left(G, \mathcal{O}_{X}\right)$ are non-uniform.

\section{$\S 3$. Non-emptiness of moduli spaces}

For a rank- 2 vector bundle $E$, we shall denote by $d_{E}$ and $r_{E}$ the invariants of $E$, when confusions may appear.

THEOREM 10. $M\left(c_{1}, c_{2}, d, r\right)$ is non-empty if and only if $l:=l\left(c_{1}, c_{2}\right.$, $d, r) \geq 0$ and one of the following conditions holds:

(I) $2 d>\alpha$ or,

(II) $2 d=\alpha, \beta-2 r \leq g+l$.

Proof. We observe that if $M \neq \emptyset$ then, by means of Theorem 1 , the elements of $M$ lie among 2-vector bundles given by extensions of type (1). Therefore, we conclude that $M \neq \emptyset$ if and only if in the extensions of type (1) there are 2-vector bundles with $d_{E}=d$ and $r_{E}=r$.

It is clear that all the vector bundles given by an extension of type (1) have $d_{E}=d$ so we shall look for bundles with $r_{E}=r$.

We fix $L_{1}, L_{2} \in \operatorname{Pic}_{0}(C)$ and $Y \subset X$ a locally complete intersection (or the empty set) and we denote

$$
\begin{aligned}
& N_{1}=\mathcal{O}_{X}\left(d^{\prime} C_{0}+s f_{0}\right) \otimes \pi^{*} L_{1} \\
& N_{2}=\mathcal{O}_{X}\left(d C_{0}+r f_{0}\right) \otimes \pi^{*} L_{2}
\end{aligned}
$$

and $l=\operatorname{deg}(Y)$.

Consider the spectral sequence of terms

$$
E_{2}^{p, q}=H^{p}\left(X, \mathcal{E} x t^{q}\left(I_{Y} \otimes N_{1}, N_{2}\right)\right)
$$

which converges to

$$
\operatorname{Ext}^{p+q}\left(I_{Y} \otimes N_{1}, N_{2}\right)
$$

We have

$$
\mathcal{E} x t^{0}\left(I_{Y} \otimes N_{1}, N_{2}\right) \cong N_{2} \otimes N_{1}^{-1} \text { and } \mathcal{E} x t^{1}\left(I_{Y} \otimes N_{1}, N_{2}\right) \cong \mathcal{O}_{Y} .
$$

But $H^{2}\left(X, N_{2} \otimes N_{1}^{-1}\right)=0$ so the exact sequence of lower terms becomes

$$
0 \rightarrow H^{1}\left(X, N_{2} \otimes N_{1}^{-1}\right) \rightarrow \operatorname{Ext}^{1}\left(I_{Y} \otimes N_{1}, N_{2}\right) \rightarrow H^{0}\left(Y, \mathcal{O}_{Y}\right) \rightarrow 0 .
$$


Now, by a result due to Serre (see [O-S-S], Chap.I, 5, [Se]), any element in the group $\operatorname{Ext}^{1}\left(I_{Y} \otimes N_{1}, N_{2}\right)$ which has an invertible image in $H^{0}\left(Y, \mathcal{O}_{Y}\right)$ defines an extension of the desired form with $E$ a 2-vector bundle.

We write the sequence (1) under the equivalent form

$$
0 \rightarrow \mathcal{O}_{X} \rightarrow E\left(-d C_{0}\right) \otimes \pi^{*} L^{\prime \prime} \rightarrow \mathcal{O}_{X}\left(\left(d^{\prime}-d\right) C_{0}+(s-r) f_{0}\right) \otimes \pi^{*}(\tilde{L}) \otimes I_{Y} \rightarrow 0
$$

where $\tilde{L}=L_{1} \otimes L_{2}^{-1}, \quad L^{\prime \prime}=\mathcal{O}_{C}(-r) \otimes L_{2}^{-1}$ and $\operatorname{deg}\left(L^{\prime \prime}\right)=-r$.

From the definition, it follows $r \leq r_{E}$ for every bundle $E$ given by an extension (1). We distinguish three cases:

(I) $d>d^{\prime}$. In this case we shall prove that $M$ is non-empty if and only if $l \geq 0$. To do this we prove that all vector bundles from extension (1) have $r_{E}=r$.

We verify that for all $L^{\prime} \in \operatorname{Pic}(C)$ with $\operatorname{deg}\left(L^{\prime}\right)<0$ we have

$$
H^{0}\left(E\left(-d C_{0}\right) \otimes \pi^{*}\left(L^{\prime \prime} \otimes L^{\prime}\right)\right)=0,
$$

which is true because $H^{0}\left(L^{\prime}\right)=0$ and

$$
H^{0}\left(\mathcal{O}_{X}\left(\left(d^{\prime}-d\right) C_{0}+(s-r) f_{0}\right) \otimes \pi^{*}\left(L_{1} \otimes L_{2}^{-1} \otimes L^{\prime}\right) \otimes I_{Y}\right)=0 .
$$

(II) $\mathrm{a}^{\circ} \cdot d=d^{\prime}, r \geq s$. Then $M$ is non-empty if and only if $l \geq 0$. The proof runs like in the first case with the remark $\operatorname{deg}\left(\mathcal{O}_{C}(s-r) \otimes L_{1} \otimes L_{2}^{-1} \otimes L^{\prime}\right)<0$. (II) $\mathrm{b}^{\circ} . d=d^{\prime}, r<s$. Then $M$ is non-empty if and only if $l \geq 0$ and $\beta-2 r \leq g+l$.

Let us see first that the natural isomorphism

$$
\begin{gathered}
M(2 d, \beta, \gamma, d, r) \longrightarrow M(0, \beta, l, 0, r) \\
E \longrightarrow E\left(-d C_{0}\right)
\end{gathered}
$$

allows us to suppose $d=d^{\prime}=0$.

In this case, the sequence (3) becomes $0 \rightarrow \mathcal{O}_{X} \rightarrow E \otimes \mathcal{O}_{X}\left(-r f_{0}\right) \otimes \pi^{*} L_{2}^{-1} \rightarrow \mathcal{O}_{X}\left((s-r) f_{0}\right) \otimes \pi^{*}\left(L_{1} \otimes L_{2}^{-1}\right) \otimes I_{Y} \rightarrow 0$.

The definition of the second invariant implies that $r_{E}=r$ if and only if $E^{\prime}:=\pi_{*} E \otimes \mathcal{O}_{C}\left(-r p_{0}\right) \otimes L_{2}^{-1}$ is normalised. $E^{\prime}$ belong to an extension

$$
0 \rightarrow \mathcal{O}_{C} \rightarrow E^{\prime} \rightarrow L \rightarrow 0
$$

where $L=\mathcal{O}_{C}\left((s-r) p_{0}\right) \otimes L_{1} \otimes L_{2}^{-1} \otimes \mathcal{O}_{C}\left(-Z_{1}\right)$ with $Z_{1}$ an effective divisor on $C$ with support $\pi(Y)$ and $\operatorname{card}(Y) \leq \operatorname{deg}\left(Z_{1}\right) \leq l=\operatorname{deg}(Y)$.

According to a result of Nagata ([N] or [Ha] Ex.V.2.5), if $E^{\prime}$ is normalised, then 


$$
-\operatorname{deg}\left(E^{\prime}\right)=r-s+\operatorname{deg}\left(Z_{1}\right) \geq-g
$$

which proves "only if" part of (II) b .

For "if" part we choose $Y$ reduced, obtained by intersection between $C_{0}$ and $l$ distinct fibres of $X$. In this case, we have the following short exact sequence

$$
0 \rightarrow I_{Z} \rightarrow I_{Y} \rightarrow I_{Y \subset Z} \rightarrow 0
$$

where $Z_{1}=\pi(Y)=p_{1}+\cdots+p_{l}, Y \subset Z=\pi^{-1}\left(Z_{1}\right)=f_{1}+\cdots+f_{l}$ with $f_{i}$ distinct fibres, $\mathcal{O}_{Z}=\mathcal{O}_{f_{1}} \oplus \cdots \oplus \mathcal{O}_{f_{l}}, I_{Y \subset Z}=\mathcal{O}_{f_{1}}(-1) \oplus \cdots \oplus \mathcal{O}_{f_{l}}(-1)$.

So, the sequence (5) becomes

$$
0 \rightarrow I_{Z} \rightarrow I_{Y} \rightarrow \mathcal{O}_{f_{1}}(-1) \oplus \cdots \oplus \mathcal{O}_{f_{l}}(-1) \rightarrow 0
$$

Tensoring by $K_{X} \otimes N_{2}^{-1} \otimes N_{1}$ and taking the long cohomology sequence we obtain an injective map:

$$
H^{1}\left(K_{X} \otimes N_{2}^{-1} \otimes N_{1} \otimes I_{Z}\right) \longrightarrow H^{1}\left(K_{X} \otimes N_{2}^{-1} \otimes N_{1} \otimes I_{Y}\right) .
$$

By dualizing, it follows that the natural map

$$
\operatorname{Ext}^{1}\left(I_{Y} \otimes N_{1}, N_{2}\right) \stackrel{\varphi}{\longrightarrow} \operatorname{Ext}^{1}\left(I_{Z} \otimes N_{1}, N_{2}\right) \cong \operatorname{Ext}^{1}\left(L, \mathcal{O}_{C}\right)
$$

is surjective, which shows that all bundles in (4) are coming from (1) by applying $\pi_{*}$.

According to $[\mathrm{Ha}]$ (Ex. V.2.5), there is a non-empty open set $V \subset$ $\operatorname{Ext}^{1}\left(L, \mathcal{O}_{C}\right)$ (don't forget the condition $s-r \leq g+l$ !) such that all $\xi \in V$ define normalised vector bundles on $C$.

Now, in $\operatorname{Ext}^{1}\left(I_{Y} \otimes N_{1}, N_{2}\right)$ the set of vector bundles is a non-empty open set $U$. It is clear that $\varphi^{-1}(V) \cap U \neq \emptyset$ (being open sets in Zariski topology), so we conclude.

\section{$\S 4$. Moduli of stable bundles}

There is an interesting relation between the moduli spaces $M\left(c_{1}, c_{2}, d, r\right)$ and the Qin's sets $E_{\zeta}\left(c_{1}, c_{2}\right)$ (see [Q1], [Q2] for precised definitions).

As in the proof of Theorem 10, case (I) we conclude that if $\zeta$ is a normalized class reprezenting a non-empty wall of type $\left(c_{1}, c_{2}\right)$ such that $l_{\zeta}\left(c_{1}, c_{2}\right)>0$ then, for $(2 d-\alpha, 2 r-\beta)=\zeta, E_{\zeta}\left(c_{1}, c_{2}\right)$ and $M\left(c_{1}, c_{2}, d, r\right)$ are coincident modulo a factor of $\operatorname{Pic}_{0}(C)$ (Qin workes with first Chern class $c_{1}$ as an element in $\operatorname{Pic}(X))$.

This is a consequence of the following facts: 
(a) $l_{\zeta}\left(c_{1}, c_{2}\right)=l\left(c_{1}, c_{2}, d, r\right)$

(b) condition $\zeta^{2}<0$ implies $2 d>\alpha$

(c) in the case $2 d>\alpha$ the bundles $L_{1}, L_{2}$ and the set $Y$ from the sequence (1) are uniquely determined by $E$.

(d) if $l\left(c_{1}, c_{2}, d, r\right)>0$ then in the sequence (1) the bundles are given only by non-trivial extensions.

In fact it is not hard to see that $M\left(c_{1}, c_{2}, d, r\right) \neq \emptyset$ iff $E_{\zeta}\left(c_{1}, c_{2}\right) \neq \emptyset$ so, by means of Theorem $10, E_{\zeta}\left(c_{1}, c_{2}\right) \neq \emptyset$ if $l_{\zeta}\left(c_{1}, c_{2}\right)>0$. But we have even more:

COROLlary 11. Let $X$ be a ruled surface different from $\mathbb{P}^{1} \times \mathbb{P}^{1}$ and let $\mathcal{C}$ be a chamber of type $\left(c_{1}, c_{2}\right)$ different from $\mathcal{C}_{f_{0}}$. Then the moduli space $\mathcal{M}_{\mathcal{C}}\left(c_{1}, c_{2}\right) \neq \emptyset$.

Proof. From Theorem 1.3.3 in [Q2] it follows that

$$
\mathcal{M}_{\mathcal{C}}\left(c_{1}, c_{2}\right)=\left(\mathcal{M}_{\mathcal{C}_{1}}\left(c_{1}, c_{2}\right)-\bigsqcup_{\zeta} E_{(-\zeta)}\left(c_{1}, c_{2}\right)\right) \bigsqcup_{\zeta} E_{\zeta}\left(c_{1}, c_{2}\right)
$$

where $\mathcal{C}_{1}$ is the chamber lying above $\mathcal{C}$ and sharing with $\mathcal{C}$ a non-empty common wall $W$ and $\zeta$ runs over all normalised classes representing $W$. By the above considerations, it follows that $E_{\zeta}\left(c_{1}, c_{2}\right) \neq \emptyset$ if $l\left(c_{1}, c_{2}, d, r\right)>0$. It remains the case $l\left(c_{1}, c_{2}, d, r\right)=0$ and it will be sufficient to prove that

$$
h^{1}\left(X, N_{2} \otimes N_{1}^{-1}\right):=\operatorname{dim} H^{1}\left(X, N_{2} \otimes N_{1}^{-1}\right)>0
$$

(see the proof of Theorem 10).

We have

$$
N_{2} \otimes N_{1}^{-1}=\mathcal{O}_{X}\left(\left(d-d^{\prime}\right) C_{0}+(r-s) f_{0}\right) \otimes \pi^{*}\left(L_{2} \otimes L_{1}^{-1}\right)
$$

where $d-d^{\prime}=2 d-\alpha=u$ and $r-s=2 r-\beta=v$. But $\zeta=u C_{0}+v f_{0}$ is a normalized class and this implies that $u>0$ and $v<0$ (see [Q1]).

Because $H^{2}\left(X, N_{2} \otimes N_{1}^{-1}\right)=0$, the Riemann-Roch Theorem gives the equality:

$\chi=h^{0}\left(X, N_{2} \otimes N_{1}^{-1}\right)-h^{1}\left(X, N_{2} \otimes N_{1}^{-1}\right)=1-g+(1 / 2)((u+1)(2 v-u e)+u(2-2 g))$.

But $\zeta^{2}<0$ gives $u(2 v-u e)<0$; it follows $2 v-u e<0$.

If $g \geq 1$, then obviously $\chi<0$. If $g=0$, then $e \geq 0$ and

$$
\chi=1+v+(u / 2)(2(v+1)-e(u+1)) .
$$


If $e \geq 1$, then $\chi<0$. For $e=0$ we get $X=\mathbb{P}^{1} \times \mathbb{P}^{1}$, which we excluded. Thus, in all cases $\chi<0$; it follows $h^{1}\left(X, N_{2} \otimes N_{1}^{-1}\right)>0$ and the proof is over.

Remark. Let us suppose that $X=\mathbb{P}^{1} \times \mathbb{P}^{1}$ and that $\mathcal{C}$ is a chamber of type $\left(c_{1}, c_{2}\right)$ lying below a non-empty wall defined by a normalized class $\zeta=u C_{0}+v f_{0}$ with $v \leq-2$. Then the same conclusion as in the above corollary holds.

Indeed, in this case we have $\chi=(1+v)(1+u)$. Since $v<-1$, then again $\chi<0$.

Acknowledgements. The second named author expresses his gratitude to the Max-Planck-Institut für Mathematik Bonn for its hospitality during the final stage of this work.

\section{REFERENCES}

[B] V. Brînzănescu, Algebraic 2-vector bundles on ruled surfaces, Ann. Univ. Ferrara-Sez VII, Sc. Mat., XXXVII (1991), 55-64.

[B-St1] V. Brînzănescu and M. Stoia, Topologically trivial algebraic 2-vector bundles on ruled surfaces I, Rev. Roumaine Math. Pures Appl., 29 (1984), 661-673.

[B-St2] Topologically trivial algebraic 2-vector bundles on ruled surfaces II, In : Lect. Notes Math., 1056, Springer (1984).

[Br1] J. E. Brossius, Rank-2 vector bundles on a ruled surface I, Math. Ann., 265 (1983), 155-168.

[Br2] — Rank-2 vector bundles on a ruled surface II, Math. Ann., 266 (1984), 199-214.

[Ha] R. Hartshorne, Algebraic Geometry, Graduate Texts in Math., 49, Springer, Berlin-Heidelberg, 1977.

[H-S] H. J. Hoppe and H. Spindler, Modulräume stabiler 2-Bündel auf Regelflächen, Math. Ann., 249 (1980), 127-140.

[K] S. Kleiman, Les théorèmes de finitude pour les Founcteurs de Picard, In : Théories des intersections et théorème de Riemann-Roch, SGA VI, Exp. XIII, Lect. Notes in Math., 225, Springer (1971), 616-666.

[N] M. Nagata, On self-intersection Number of a section on ruled surface, Nagoya Math. J., 37 (1970), 191-196.

[O-S-S] C. Okonek, M. Schneider and H. Spindler, Vector bundles on complex projective spaces, Birkhäuser, Basel Boston Stuttgart, 1980.

[Q1] Z. Qin, Moduli spaces of stable rank-2 bundles on ruled surfaces, Invent. Math., 110 (1992), 615-626.

[Q2] Equivalence classes of polarizations and moduli spaces of sheaves, J. Diff. Geom., 37 (1993), 397-415. 
[Se] J. P. Serre, Sur les modules projectifs, Sém. Dubreil-Pisot 1960/1961 Exp. 2, Fac. Sci. Paris, 1963.

[T1] F. Takemoto, Stable vector bundles on algebraic surfaces I, Nagoya Math. J., 47 (1972), 29-48.

[T2] W Stable vector bundles on algebraic surfaces II, Nagoya Math. J., 52 (1973), 173-195.

[W] C. H. Walter, Components of the stack of torsion-free sheaves of rank-2 on ruled surfaces, Math. Ann., 301 (1995), 699-716.

Marian Aprodu

Institute of Mathematics of the Romanian Academy

P.O. BOX 1-764, RO-70700 Bucharest

Romania

aprodu@stoilow.imar.ro

Vasile Brînzănescu

Institute of Mathematics of the Romanian Academy

P.O. BOX 1-764, RO-70700 Bucharest

Romania

brinzane@stoilow.imar.ro 